levels. We aimed to identify review level evidence on interventions to address or prevent gambling related harm, to document the scope and nature of the evidence base, any gaps in the evidence, and identify policy implications, including where further development and evaluation of interventions was most urgently needed to support the development of evidenceinformed policy.

Methods Systematic mapping review and narrative synthesis of review level evidence for any intervention that aimed to address or prevent gambling related harm. Inclusion criteria included all forms of gambling; all populations (whole population; identified/self-defined gamblers and specific populations at risk (e.g. children and young people); all reported outcome measures.

Results After duplication, the searches generated 1080 records. Of 43 potential papers, 13 were excluded at the full paper stage and 30 papers were included in the review. We identified seven studies relating to whole population preventative interventions (demand reduction interventions to reduce the demand for gambling $(n=3)$, and supply reduction interventions to limit opportunities to gamble $(n=4))$. Twenty three studies provided evidence relating to targeted treatment interventions for individuals with an identified gambling addiction (therapeutic interventions $(n=12)$, pharmacological interventions $(n=4)$, self-help/ mutual support interventions $(n=5)$, and studies comparing two or more of these approaches $(n=2))$. Two further types of intervention we had expected to find were not represented in the systematic review level evidence. These were interventions to screen, identify and support individuals at risk of gambling related harm (whole population) and interventions to support ongoing recovery and prevent relapse into gambling related harm for individuals with an identified gambling addiction.

Conclusion A public health approach suggests that to reduce gambling related harm, there is potential to deliver interventions across the whole pathway - from population-level regulation of provision of opportunity to gamble to screening to identify those at risk, to targeted services for populations with an identified problem already causing harms. Whilst there is some evidence for downstream interventions, there is a dearth of evidence for wider public policy interventions suggesting that implementation of these must be accompanied by robust evaluation of effectiveness.

\section{P87 ARE PEOPLE WHO EXPERIENCE HARM FROM OTHERS' DRINKING ALCOHOL MORE LIKELY TO SMOKE? A CROSS SECTIONAL ANALYSIS OF NATIONAL SURVEY DATA}

JB Burton, MH Horton* ${ }^{*}$ CG Griffiths. Health Improvement, Public Health England, London, UK

\subsection{6/jech-2020-SSMabstracts. 179}

Background Smoking is a modifiable risk factor and has a well-known relationship with socioeconomic status and mental health. However, research into alcohol harm from others and its association with smoking habits is sparse. Despite a significant minority $(20.1 \%)$ of the population experiencing some form of harm from other's alcohol consumption there has been little study into its effects on smoking habits. In this study we aimed to compare the prevalence of smoking in those who experience harm from other's alcohol consumption and those who don't.

Methods We conducted cross-sectional analysis on the Alcohol Toolkit Study data from 2015 to 2016. Households were selected using a hybrid system of random probability sampling and simple quota sampling. Respondents aged over 16 were asked about their smoking habits and whether they had experienced harm from another's drinking in the last year. Aggressive harm was categorised to include more serious harms. We used logistic regression to explore if the characteristics of those who experienced harm including age, ethnicity, work status, disability, housing tenure, life stage, qualifications and personal drinking, account for any of the difference in smoking rates between the people who experience harm and those who don't.

Results 4881 participants were included in the analysis. 38\% of respondents that had experienced aggressive harm from others' alcohol consumption were smokers compared with $26 \%$ of those who had experienced some harm and $17 \%$ of those who had experienced no harm. Some of the variation was explained by the respondents' personal characteristics, but the odds of smoking remained higher for those who experienced harm (OR $1.2795 \mathrm{CI} \%$ 1.05-1.56) and for those who experienced aggressive harm (OR 1.92 95\%CI 1.46-2.53) compared with those who did not.

Conclusion Alcohol and tobacco use are closely linked. This exploratory analysis has allowed us to show that the link extends to people who have experienced harm from another person's drinking. People who have experienced harm are more likely to smoke, particularly for those who experience aggressive harm. The reasons for this correlation between alcohol harm and tobacco use have not been investigated before and this study shows that the relationship does not appear solely to be due to sociodemographic characteristics. This warrants further study. Smoking cessation services should take into consideration the impacts of harm from other's alcohol consumption on the smokers attempts to quit.

\section{P88 EXPLORING THE RELATIONSHIP BETWEEN SOCIAL MEDIA USE AND ALCOHOL CONSUMPTION OVER TIME AMONG YOUTHS; FINDINGS FROM UNDERSTANDING SOCIETY}

L Ng Fat* ${ }^{*}$ N Cable. Epidemiology and Public Health, University College London, London UK

\subsection{6/jech-2020-SSMabstracts. 180}

Background Given the decline in alcohol consumption amongst young people, which has coincided with the rise in technological use, there is a need for research to investigate whether technological use might be impacting on how young people drink alcohol. This study explores how social media use, and changes in social media use over time might affect alcohol consumption among youths.

Methods Using data from Understanding Standing, a UK household longitudinal study, from participants aged 12-19 $(\mathrm{N}=3586)$ from the youth and main survey in Wave 3 (2011-2013), and followed up in Wave 6 (2014-2016), we explored whether belonging to social media sites and frequency of interaction on social media sites in the past week, were related to being a drinker and drinking 
frequency in the past four weeks respectively, at baseline. Calculating change scores over time, we assessed whether changes in social media belonging, were associated with changes in drinking status, and whether changes in frequency of use of social media sites were related to changes in drinking frequency, using multinomial logistic and linear regression model. Analyses were repeated with number of times binge drinking $(5+$ drinks $)$ in the past four weeks, on a sub-sample of participants aged 16-19. Models adjusted for sex, age, household tenure, urban/rural area, educational/work status, baseline social media use/frequency and number of friends.

Results At baseline, those who belonged to a social media site were over four times more likely $(\mathrm{OR}=4.6295 \% \mathrm{CI}$ (3.61-5.91)) to be a drinker versus non-drinker. Those who chatted for longer on social media sites were more likely to have drank at least once a week compared with not drinking in the past month (none vs. 1-3 hours (2.31(1.45$3.68)$, 4-6 hours 2.60(1.44-4.72), 7 hours or more 5.92 (2.86-12.28). Among participants aged 16-19, a similar gradient was found with higher odds of binge drinking three or more times in the past month, with greater chat frequency (e.g. no hours chatting vs. 7 hours or more 6.29 (2.50-15.82)). Over time, becoming new social media users increased the odds of becoming a new drinker (1.77 (1.043.03)) versus no change in drinking status. Participants who became more frequent users of social media sites, were more likely to drink more frequently $(\beta=0.13,95 \%$ CI $(0.09-0.17))$, and binge drink $(\beta=0.21$, 95\% CI $(0.12-$ $0.31)$ ) more frequently across waves.

Conclusion Increasing social media use was associated with greater alcohol consumption; mechanisms behind this relationship should be investigated. Alcohol-related content on the internet should be monitored.

\section{P89 TACKLING LOCAL ALCOHOL-RELATED HARMS IN THE NIGHT TIME ECONOMY: A PROCESS EVALUATION WITH A COMPLEX SYSTEMS PERSPECTIVE}

${ }^{1} \mathrm{ET}$ McGill*, ${ }^{2} \mathrm{D}$ Marks, ${ }^{3} \mathrm{M}$ Petticrew, ${ }^{3} \mathrm{M}$ Egan. ${ }^{1}$ Deaprtment of Health Services, Research and Policy, London School of Hygiene and Tropical Medicine, London, UK; ${ }^{2}$ Department of Public Health, Environments and Society, London School of Hygiene and Tropical Medicine, London, UK

\subsection{6/jech-2020-SSMabstracts. 181}

Background English local authorities (LAs) have certain powers they can utilise to help them tackle alcohol-related harms. One such power is the Late Night Levy (LNL) which uses a 'polluter pays' principle whereby the LA charges alcohol retailers a fee for selling alcohol after midnight. Public health evaluators are increasingly considering how concepts from complexity science and systems thinking might shed light on how interventions can affect health in complex, real world settings such as local alcohol systems. This study aims to explore how the LNL acts as an event within the alcohol system, with a particular emphasis on understanding how the intervention, actors and the system as a whole adapt and coevolve in response.

Methods We conducted a process evaluation, using a complex systems perspective, in one LA. We included a range of system actors and utilised qualitative data generation methods. We interviewed LNL implementers and alcohol consumers $(n=21)$, conducted observations in pubs and during community-safety patrols (34.5 hours) and reviewed documents on the LNL and the broader system $(n=15)$. We conducted a deductive thematic analysis, applying concepts from systems thinking and complexity science to: 1) describe the system into which the LNL is introduced; and 2) identify emergent system outcomes.

Results The LNL operates within a complex system consisting of diverse actors with competing goals; these include the local political system and statutory functions, the retail sector, the third sector, residents and users of the nighttime economy. Early analyses identified a number of system responses stemming from the intervention, which include: 1) retailer adaptation to the levy; 2) new and evolving relationships between retailers, the police, community-safety officers and LA officers; 3) changes in venue management; and 4) the evolution of the intervention itself in response to broader system feedback. These adaptations are hypothesised to affect alcohol-related health and social harms. However, the intervention also interacts with wider system influences on public health, for example local government budgetary constraints during a period of central government cuts.

Discussion Conceptualising the LNL as an event within a complex system allows us to analyse its trajectory as it embeds within, and influences the broader system in which it is located. Further fieldwork and analyses are ongoing and focus on sampling additional system actors and exploring the influence of the vertical systems (e.g. national policy) which shape local responses to alcohol-related harms.

This study is funded by the National Institute for Health Research (NIHR) School for Public Health Research (Grant Reference Number PD-SPH-2015). The views expressed are those of the author(s) and not necessarily those of the NIHR or the Department of Health and Social Care.

\section{P90 HEALTH-JUSTICE PARTNERSHIPS: A SYSTEMATIC SCOPING REVIEW OF INTERNATIONAL EVIDENCE ON IMPACT}

${ }^{1} \mathrm{~S}$ Beardon*, ${ }^{2} \mathrm{C}$ Woodhead, ${ }^{1} \mathrm{~S}$ Cooper, ${ }^{3} \mathrm{H}$ Genn, ${ }^{1} \mathrm{R}$ Raine. ${ }^{1}$ Department for Applied Health Research, University College London, London, UK; ${ }^{2}$ Department of Psychological Medicine, King's College London, London, UK; ${ }^{3}$ Faculty of Laws, University College London, London, UK

\subsection{6/jech-2020-SSMabstracts. 182}

Background Social welfare legal problems impact negatively on mental and physical health. These include issues with welfare benefits, debt, homelessness, family and employment. Services providing legal assistance have developed collaborations with healthcare across the UK and the globe. These 'health-justice partnerships' aim to support health of the most disadvantaged groups, tackling interconnected challenges of health inequalities and access to justice. International evidence on the impacts of these partnerships has never previously been reviewed. This study interrogates the state of current evidence and provides new insights from international practice.

Methods A systematic scoping review of international literature was undertaken. Both academic and grey literature was included from OECD countries, 1995-2018. The review 